\title{
Posttraumatic Stress Disorder Is Associated with $\alpha$ Dysrhythmia across the Visual Cortex and the Default Mode Network
}

\author{
DKevin J. Clancy, ${ }^{1}$ Jeremy A. Andrzejewski, ${ }^{1}$ Jessica Simon, ${ }^{1}$ Mingzhou Ding, ${ }^{2}$ Norman B. Schmidt, ${ }^{1}$ \\ and ${ }^{-W}$ Wen $\mathrm{Li}^{1}$
}

https://doi.org/10.1523/ENEURO.0053-20.2020

${ }^{1}$ Department of Psychology, Florida State University, Tallahassee, FL 32304 and ${ }^{2} \mathrm{~J}$. Crayton Pruitt Family Department of Biomedical Engineering, University of Florida, Gainesville, FL 32611

\begin{abstract}
Anomalies in default mode network (DMN) activity and $\alpha(8-12 \mathrm{~Hz})$ oscillations have been independently observed in posttraumatic stress disorder (PTSD). Recent spatiotemporal analyses suggest that $\alpha$ oscillations support DMN functioning via interregional synchronization and sensory cortical inhibition. Therefore, we examined a unifying pathology of $\alpha$ deficits in the visual-cortex-DMN system in PTSD. Human patients with PTSD $(N=25)$ and two control groups, patients with generalized anxiety disorder (GAD; $N=24$ ) and healthy controls ( $H C s ; N=20$ ), underwent a standard eyes-open resting state (S-RS) and a modified resting state (M-RS) of passively viewing salient images (known to deactivate the DMN). High-density electroencephalogram (hdEEG) were recorded, from which intracortical $\alpha$ activity (power and connectivity/Granger causality) was extracted using the exact low-resolution electromagnetic tomography (eLORETA). Patients with PTSD (vs GAD/HC) demonstrated attenuated $\alpha$ power in the visual cortex (VC) and key hubs of the DMN [posterior cingulate cortex (PCC) and medial prefrontal cortex (mPFC)] at both states, the severity of which further correlated with hypervigilance symptoms. With increased visual input (at M-RS vs S-RS), patients with PTSD further demonstrated reduced $\alpha$-frequency directed connectivity within the DMN (PCC $\rightarrow \mathrm{mPFC})$ and, importantly, from the $\mathrm{VC}$ to both $\mathrm{DMN}$ hubs $\mathrm{VC} \rightarrow \mathrm{PCC}$ and $\mathrm{VC} \rightarrow \mathrm{mPFC})$, linking $\alpha$ deficits in the two systems. These interrelated $\alpha$ deficits align with DMN hypoactivity/hypoconnectivity, sensory disinhibition, and hypervigilance in PTSD, representing a unifying neural underpinning of these anomalies. The identification of visual-cortex-DMN $\alpha$ dysrhythmia in PTSD further presents a novel therapeutic target, promoting network-based intervention of neural oscillations.
\end{abstract}

Key words: $\alpha$ oscillations; default mode network; posttraumatic stress disorder; resting state; sensory disinhibition

\section{Significance Statement}

$\alpha(8-12 \mathrm{~Hz})$ oscillations and the default mode network (DMN) both dominate the resting-state brain activity and are found to be closely related. In addition, aberrant $\alpha$ and DMN activities are both implicated in the pathophysiology of posttraumatic stress disorder (PTSD). Linking $\alpha$ and DMN aberrations in PTSD, our high-density electroencephalogram (hdEEG) source analysis reveals that PTSD is associated with $\alpha$ power deficits across the DMN and visual cortex (VC) and deficient $\alpha$-frequency connectivity from the VC to the DMN. That this visual-cortex-DMN $\alpha$ dysrhythmia further underpins hypervigilance symptoms in PTSD highlights a temporal-spatial network pathology, promoting network-based neural oscillatory interventions.

Received February 13, 2020; accepted June 19, 2020; First published July 20, 2020.

The authors declare no competing financial interests.
Author contributions: K.J.C. and N.B.S. designed research; K.J.C., J.S., and W.L. performed research; M.D. contributed unpublished reagents/analytic tools; K.J.C., J.A.A., J.S., M.D., and W.L. analyzed data; K.J.C., J.A.A., J.S., N.B.S., and W.L. wrote the paper. 


\section{Introduction}

Prevailing models of the neuropathology of posttraumatic stress disorder (PTSD) have focused on dysfunctions of the prefrontal-cortex-amygdala circuit (Rauch et al., 2006; Liberzon and Abelson, 2016). Recently, evidence has extended this circuit pathology to implicate large-scale brain network anomalies (Spielberg et al., 2015; Koch et al., 2016; Liberzon and Abelson, 2016; Akiki et al., 2017). Anomalies in the default mode network (DMN), a major resting-state network (RSN), have been especially highlighted in this literature, characterized by attenuated network activity and disrupted network communication (Bluhm et al., 2009; Lanius et al., 2010; Sripada et al., 2012; Koch et al., 2016; Akiki et al., 2018).

The DMN is one of the most consistently identified RSNs, anchored in a midline core consisting of two key hub structures, the posterior cingulate cortex (PCC) and the medial prefrontal cortex (mPFC; Buckner et al., 2008; Yeo et al., 2011). While prominent in the resting state, the DMN is deactivated by salient sensory input or externallyoriented cognitive processing and, accordingly, exhibits reciprocal inhibition with neural networks associated with these processes (Gusnard et al., 2001; Raichle et al., 2001; Greicius et al., 2003; Greicius and Menon, 2004). As such, DMN dysfunctions can interrupt internal mentation or 'tranquil' resting states and heighten vigilance and attention. Indeed, DMN dysfunction has been linked to heightened acute stress response (Menon, 2011; Hermans et al., 2014; Zhang et al., 2019) and implicated in PTSD symptoms of hypervigilance and negative intrusions (Abdallah et al., 2017; Akiki et al., 2018).

While neuroimaging data have isolated the DMN as the dominant network in the resting brain, electrophysiological data have identified $\alpha(8-12 \mathrm{~Hz})$ oscillations as the dominant electrical activity in the resting brain (Klimesch et al., 2007; Klimesch, 2012). $\alpha$ Oscillations represent a neural mechanism mediating long-range interregional interactions (Palva and Palva, 2007; Tang et al., 2007; Hillebrand et al., 2016). Importantly, evidence has begun to link $\alpha$ oscillations to $\mathrm{DMN}$ activity (especially during eyes-open resting state; Mantini et al., 2007; Jann et al., 2009; Knyazev et al., 2011; Scheeringa et al., 2012; Mo et al., 2013), raising the possibility that, to some extent, the DMN could be organized and maintained by long-range synchronization of $\alpha$ oscillations (Engel and Singer, 2001; Uhlhaas et al., 2008; Jann et al., 2009).

While $\alpha$ oscillations positively correlate with DMN activity, they are known to negatively correlate with visual

This work was supported by the National Institute of Mental Health Grant R01MH093413 (to W.L.), the Florida State University Chemical Senses Training Grant Award T32DC000044 (to K.J.C.) from the National Institutes of Health/National Institute on Deafness and Other Communication, and the Subaward of United States Army Award W81XWH-10-2-018 (to N.B.S.).

Correspondence should be addressed to Wen Li at wenli@psy.fsu.edu or Kevin J. Clancy at clancy@psy.fsu.edu.

https://doi.org/10.1523/ENEURO.0053-20.2020

Copyright (C) 2020 Clancy et al.

This is an open-access article distributed under the terms of the Creative Commons Attribution 4.0 International license, which permits unrestricted use, distribution and reproduction in any medium provided that the original work is properly attributed. cortical activity (Klimesch et al., 2007; Jensen and Mazaheri, 2010; Lange et al., 2013). $\alpha$ Oscillations (originating in the sensory cortex and thalamus) play a key role in visual inhibition by suppressing cortical excitation and feedforward propagations (Klimesch et al., 2007; Palva and Palva, 2007; Tang et al., 2007; Jensen and Mazaheri, 2010; Foxe and Snyder, 2011; Klimesch, 2012; Hillebrand et al., 2016; Johnson et al., 2017). This active function of $\alpha$ oscillations can present a second mechanism, visual cortical inhibition, to support DMN activity. That is, by suppressing visual processing, $\alpha$ oscillations could protect the DMN from environmental disruptions.

Alternatively, deficient $\alpha$ activity could give rise to $\mathrm{DMN}$ dysfunctions by failing to sustain long-range synchronization across the DMN and failing to inhibit sensory afferents to the DMN. Aberrant $\alpha$ oscillations have been featured in a "thalamocortical dysrhythmia" model of neuropsychiatric disorders (Llinás et al., 1999; Schulman et al., 2011), which are conceptualized transdiagnostically as oscillopathies (Basar, 2013; Buzsáki et al., 2013). Reduced resting-state $\alpha$ activity has been observed in patients with PTSD [vs healthy controls $(\mathrm{HCs})$ and patients with generalized anxiety disorder (GAD); Clancy et al., 2017] and combat veterans with severe symptoms (Clancy et al., 2020). Given the demonstrated association between $\alpha$ oscillations and DMN activity, we hypothesized that PTSD is associated with deficient $\alpha$ activity in the DMN and deficient inhibition of sensory cortical input to the DMN.

Therefore, by extracting intracortical $\alpha$ activity from high-density electroencephalogram (hdEEG) recordings, we conducted source-level analysis of resting-state $\alpha$ activity in patients with PTSD, relative to $\mathrm{HCs}$ and patients with GAD. The GAD group was included to rule out effects of general anxiety and hyperarousal that would confound resting-state neural oscillations (Imperatori et al., 2019). For simplicity and statistical rigor, the GAD group and HC group were collapsed into a single control group, which was further justified by prior work by (Clancy et al. (2017), demonstrating a lack of surface-level differences between $\mathrm{HC}$ and GAD groups. Comparisons between PTSD and HC or GAD groups separately are also reported. To highlight the vulnerability of the DMN to sensory input, we included a modified resting state (M-RS) involving passive viewing of images, in addition to a standard, eyes-open resting state (S-RS). We assessed two specific hypotheses of $\alpha$ deficits in PTSD, including (1) attenuated $\alpha$ power and connectivity in the DMN and (2) a deficit of $\alpha$ inhibition of visual cortical (VC) activity (i.e., attenuated $\alpha$ power) and attenuated directed $\alpha$-frequency connectivity to the DMN (VC $\rightarrow$ DMN). Finally, we hypothesized that deficient sensory inhibition could be associated with the PTSD symptom of hypervigilance (characterized by excessive sensory scanning of the environment for threat) and thus examined these clinical associations, linking neuropathology to clinical symptomatology.

\section{Materials and Methods}

\section{Participants}

Participants consisted of outpatients with a current diagnosis of PTSD $(N=25)$ or GAD $(N=24)$, and HCs 
$(N=20)$ with no current or past-year diagnoses. Participants were matched for age and gender across groups. Participants were recruited through community advertisement as part of a larger randomized controlled trial and compensated monetarily for their participation in the study. Inclusion criteria were primary diagnoses of PTSD or GAD, respectively, or no current or past-year psychiatric disorders for the HC group. Participants with co-morbid diagnoses of PTSD and GAD were excluded. Participants were further excluded based on the following criteria: history of suspected severe TB1 (i.e., exceeding $30 \mathrm{~min}$ of loss of consciousness because of a head or neck injury), history of neurologic disorders, and diagnosis of psychotic disorders, severe substance use disorder, or abuse of opioids, stimulants, or cocaine. Six PTSD participants met diagnostic criteria for mild alcohol use disorder $(n=3)$ or mild cannabis use disorder $(n=3)$; $32 \%$ of controls (GAD $n=7, \mathrm{HC} n=7$ ) had a history of a DSM- 5 criterion A trauma. Index trauma types of participants with PTSD ranged from combat exposure $(n=6)$ and vehicular accident $(n=3)$ to rape $(n=7)$ and sexual $(n=5)$ or physical assault $(n=4)$; $48 \%(n=12)$ of participants in the PTSD group reported multiple traumas. The mean number of traumas in the PTSD group was 4.22 ( \pm 2.26$)$. All participants provided written, informed consent to participate in the study, which was approved by both the university's Institutional Review Board and the Department of Defense Human Research Protection Official's Review. Demographic details are presented in Table 1.

\section{Clinical assessment}

Current or past-year diagnoses were assessed by trained clinicians using the Structured Clinical Interview for DSM-5 (American Psychiatric Association, 2013). Participants additionally completed the PTSD Checklist for DSM-IV: civilian version (Blanchard et al., 1996). In the present study, internal consistency was high for the total questionnaire $(\alpha=0.95)$ and the hyperarousal subscale $(\alpha$ $=0.84$ ). As in previous work (Mueller-Pfeiffer et al., 2013; Clancy et al., 2017), item 16 assessing symptoms of hypervigilance or "being watchful or on guard" was extracted to index hypervigilance.

\section{Experimental paradigm}

Participants were seated in a comfortable recliner in a dimly lit, sound attenuated and electrically shielded room. hdEEG data were recorded during two resting states. To evaluate intrinsic neural activity, a standard resting state (S-RS) recording was conducted first, lasting 2 min while participants fixated on a crosshair on the screen. To assess the impact of environmental sensory input on $\alpha$ oscillations and DMN functioning, a M-RS recording was also conducted, involving $5 \mathrm{~min}$ of passively viewing a continuous stream of images (subtending a visual area of $7.8^{\circ} \times 5.8^{\circ}$ ), each for $333 \mathrm{~ms}$. Images were chosen from the International Affective Picture System (Lang et al., 2008), depicting neutral (e.g., buildings, daily objects; $n=322$ ), positive (e.g., erotic; $n=253$ ), and negative (e.g., mutilation; $n=346$ ) scenes, randomly intermixed.
Table 1: Participant demographics

\begin{tabular}{lll}
\hline & PTSD & Controls \\
\hline Age (years) & $34.6 \pm 10.4$ & $31.1 \pm 13.1$ \\
Gender (female/male) & $16 / 9$ & $25 / 19$ \\
Substance use (\%) & $64 \%^{*}$ & $7.5 \%$ \\
Medication use (\%) & $40 \%$ & $36 \%$ \\
PCL total & $61.0 \pm 16.1^{*}$ & $37.6 \pm 13.4$ \\
PCL-hypervigilance & $4.1 \pm 1.0^{*}$ & $2.1 \pm 1.2$ \\
BAl & $26.2 \pm 15.7^{*}$ & $11.8 \pm 9.4$ \\
BDI & $26.8 \pm 12.5^{*}$ & $17.4 \pm 9.7$ \\
\hline
\end{tabular}

$\mathrm{PCL}=$ posttraumatic stress disorder checklist; $\mathrm{BAI}=$ Beck anxiety inventory; $\mathrm{BDI}=$ Beck depression inventory .

${ }^{+}=$Subjects with opioid, stimulant, and cocaine use were excluded. $* p<0.005$.

\section{EEG acquisition and preprocessing}

EEG data were recorded from a 96 channel BrainProducts actiCap system with Neuroscan SynAmps RT amplifiers (1000- $\mathrm{Hz}$ sampling rate, $0.05-$ to $200-\mathrm{Hz}$ online bandpass filter, referenced to the $\mathrm{FCz}$ channel). Electrooculogram (EOG) was recorded using four electrodes with vertical and horizontal bipolar derivations. EEG/EOG data were downsampled to $250 \mathrm{~Hz}$, high-pass $(1 \mathrm{~Hz})$ and notch $(60 \mathrm{~Hz})$ filtered. We then applied Fully Automated Statistical Thresholding for EEG artifact Rejection algorithm (FASTER; Nolan et al., 2010) for artifact detection, correction, and rejection. Output data were epoched into 1-s segments and submitted to eLORETA for source analyses.

\section{Exact low-resolution electromagnetic tomography (eLORETA)}

Using the high-density, artifact-minimized EEG data, we conducted intracranial source analyses using eLORETA, a linear inverse solution to reconstruct cortical activity with scalp EEG data (Pascual-Marqui et al., 2011). The LORETA algorithm has been cross-validated in multiple studies combining EEG-based LORETA with fMRI (Worrell et al., 2000; Vitacco et al., 2002; Mulert et al., 2004; Mobascher et al., 2009; Olbrich et al., 2009), positron emission tomography (Dierks et al., 2000; Pizzagalli et al., 2004), and intracranial recordings (Zumsteg et al., 2005). The solution space consists of 6239 cortical gray matter voxels with a spatial resolution of $5 \times 5 \times 5 \mathrm{~mm}$ in a realistic head model. eLORETA is a suitable tool to investigate network activity and connectivity (Neuner et al., 2014; Thatcher et al., 2014; Liu et al., 2017; Samogin et al., 2019) and has provided important network insights into psychiatric disorders (Whitton et al., 2018; Imperatori et al., 2019; Samogin et al., 2019).

For accurate inverse solutions, eLORETA was modeled for the S-RS and M-RS separately, from which wholebrain source estimates of $\alpha(8-12 \mathrm{~Hz})$ power were derived (Pascual-Marqui et al., 2011). For source-based $\alpha$-frequency connectivity analysis, time series of regions of interest (ROls) were derived from eLORETA (Samogin et al., 2019), which were then submitted to Granger causality analysis based on bivariate autoregressive (AR) modeling (Ding et al., 2006). A model order of 20 (80 ms in time for a sampling rate of $250 \mathrm{~Hz}$ ) was chosen in a two-step process: (1) Akaike Information Criterion (AIC) and (2) comparing 
spectral estimates obtained by the Fourier-based AR model for data pooled across all subjects (Wang et al., 2016). Our focus on the $\alpha$ frequency was guided by a priori hypotheses stemming from (1) the purported role of $\alpha$ oscillations in the sensory disinhibition model of PTSD and (2) prior work by Clancy et al. (2017), demonstrating no surface-level effects of PTSD-related aberrations in neighboring $\theta$ or $\beta$ frequencies ( $p s>0.16$ ).

For ROIs, DMN hubs (i.e., PCC and mPFC) and visual cortex (VC) were selected as ROls (Yeo et al., 2011; Tamber-Rosenau et al., 2013). ROls were defined by gray-matter voxels within a $10-\mathrm{mm}$ radius of the $\mathrm{ROI}$ centroids (Pascual-Marqui et al., 2011; Whitton et al., 2018). All ROls were centered on the midline to incorporate both hemispheres, with centroid coordinates obtained from the Neurosynth (https://www.neurosynth.org) meta-analysis maps (as peak voxels) of "default mode" (for the PCC: 0, $-50,30$ and the mPFC: 0, 50, 0) and "passive viewing" (for the VC: 0, -90, 20). All coordinates are reported in Montreal Neurologic Institute (MNI) space. For connectivity analysis, both directions for each pair were examined such that the three ROls resulted in a $3 \times 3$ matrix of $\alpha$-GC connectivity for each of the two resting states.

\section{Statistical analyses}

Source-level power and GC were submitted to planned simple contrasts of states (S-RS vs M-RS) to demonstrate the extent of $\alpha$ adaption from S-RS to M-RS. We then performed simple contrasts of group (PTSD vs control) for the two states to test PTSD-related deficits in $\alpha$ power and GC, and double contrasts of state and group to assess the effect of visual stimulation (S-RS minus M-RS) between groups. Pearson correlations were performed to assess clinical associations of $\alpha$ power and GC with symptom severity of hypervigilance. Guided by previous surface-level analyses showing no difference between the two control groups (GAD and HC; Clancy et al., 2017), we combined them into a single control group. Our supplemental analyses confirmed that these two control groups did not differ in source-level $\alpha$ activity.

Multiple comparison corrections were applied for the analyses. For power analyses involving whole-brain voxel-wise comparisons, we used Monte Carlo simulations (with actual Gaussian filter widths extracted from the data) to derive the corrected threshold $(p<0.05)$ : voxel level $p<0.005$ (one-tailed) over 11 contiguous voxels. As for connectivity analyses, the $3 \times 3$ connectivity matrix resulted in six comparisons for each hypothesis testing, for which we applied the false discovery rate (FDR) criterion (FDR $p<0.05$ ). Lastly, for clinical association analyses, we conducted confirmatory correlation analyses constrained to regions demonstrating main effects, for which correction was not applied. We also applied a wholebrain regression of $\alpha$ power on hypervigilance scores, followed by Monte Carlo multiple comparison correction. While the sensory hypothesis implicates a direct association between sensory disinhibition and hypervigilance symptoms, it is possible that this sensory pathology also contributes to other PTSD symptom clusters. However, correlations between reduced source-level $\alpha$ power and total PCL scores (S-RS/M-RS $r s>-0.10 /-0.14, p s>$ $0.41 / 0.25$ ) or other subscale scores ( $r s>-0.16$; $p s>0.19)$ indicate rather weak effects. We thus focused on the hypervigilance symptoms below. Trend-level and non-corrected effects will be reported but not further discussed.

\section{Results}

\section{PTSD-related $\alpha$ power deficits in the DMN and VC}

Validating intracranial source estimation of $\alpha$ oscillations, we observed " $\alpha$ blocking" by visual stimulation. Specifically, the contrast between S-RS versus M-RS (collapsed across groups) isolated $\alpha$ power reduction at M-RS across a large cluster (624 voxels) spanning bilateral visual cortices (peak voxel: $-20,-100,0 ; t=-4.50$, $d=1.09$ ).

Next, simple contrasts of group (PTSD vs controls) revealed $\alpha$ power deficits in PTSD across the visual and DMN ROls at each state. The VC exhibited the strongest group effects: at S-RS, a large cluster (283 voxels) spanning bilateral cuneus (peaks: $5,-70,30 /-5,-70,30$; ts < -3.41 , ds $>0.83$ ), bilateral precuneus (peaks: $5,-60,35 /$ $-5,-65,25$; ts $<-3.75, d s>0.92)$, and the right superior occipital gyrus (peak: $40,-80,25 ; t=-3.90$, $d s>0.96$; Fig. $1 A$ ); at M-RS, a cluster (68 voxels) in the bilateral precuneus, overlapping the S-RS cluster (peaks: 5, -55, 50/ $-5,-55,40$; ts $<-3.11$, ds $>0.76$; Fig. 1B). Regarding the DMN, $\alpha$ deficits emerged in PTSD in the bilateral PCC for both states (S-RS: 60 voxels; peaks: 15, -45, 40/-5, $-50,35$; ts < -3.32, ds > 0.81; M-RS: 199 voxels; peaks: $5,-30,25 /-5,-30,25$; ts $<-3.76$, ds $>0.92$; Fig. 1A,B), and the bilateral mPFC at M-RS only (88 voxels; peaks: 15, 60, -10/-15, 65, -15; ts < -3.10, ds 0.76; Fig. 1B). Additional $\alpha$ power deficits (whole-brain corrected) appeared in the bilateral insula [S-RS: right/left: 49/76 voxels; peaks: 35, -5, 20/-35, 20, 5 (Fig. 1A); M-RS: right/left: 136/ 25 voxels; peaks: 40, -5, 15/-30, 25, 5 (Fig. 1B); ts < -3.52 , ds $=0.86]$. No significant clusters emerged for enhanced $\alpha$ power in the PTSD group, even at a lenient threshold of $p<0.05$. Finally, double contrasts of state (M-RS minus S-RS) and group (PTSD minus control) yielded no difference between groups (voxel-level $p s>0.11$ ). These group effects are summarized in Table 2.

Individual group contrasts of PTSD versus GAD or HC separately were then performed to substantiate the PTSD versus controls difference. As summarized in Table 3, concerning $\alpha$ power, these contrasts revealed essentially identical results as reported above. Moreover, the effect sizes were comparable $(d s=0.74-1.04)$ and survived correction for multiple comparisons. In addition, $\mathrm{HC}$ and GAD did not differ on any of these contrasts (ps $>0.39$ ).

\section{PTSD-related $\alpha$ connectivity deficits within and between the DMN and VC}

Like the power analyses above, we first assessed the effect of State (S-RS vs M-RS) on $\alpha$-frequency connectivity (collapsed across the groups). We observed reduced bidirectional $\alpha$ connectivity from the S-RS to the M-RS within the DMN: $\mathrm{PCC} \rightarrow \mathrm{mPFC}(\mathrm{M}-\mathrm{RS}$ minus S-RS; $t=-3.49$, 
A
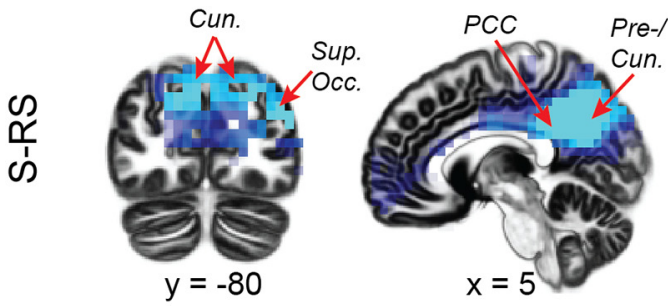

B
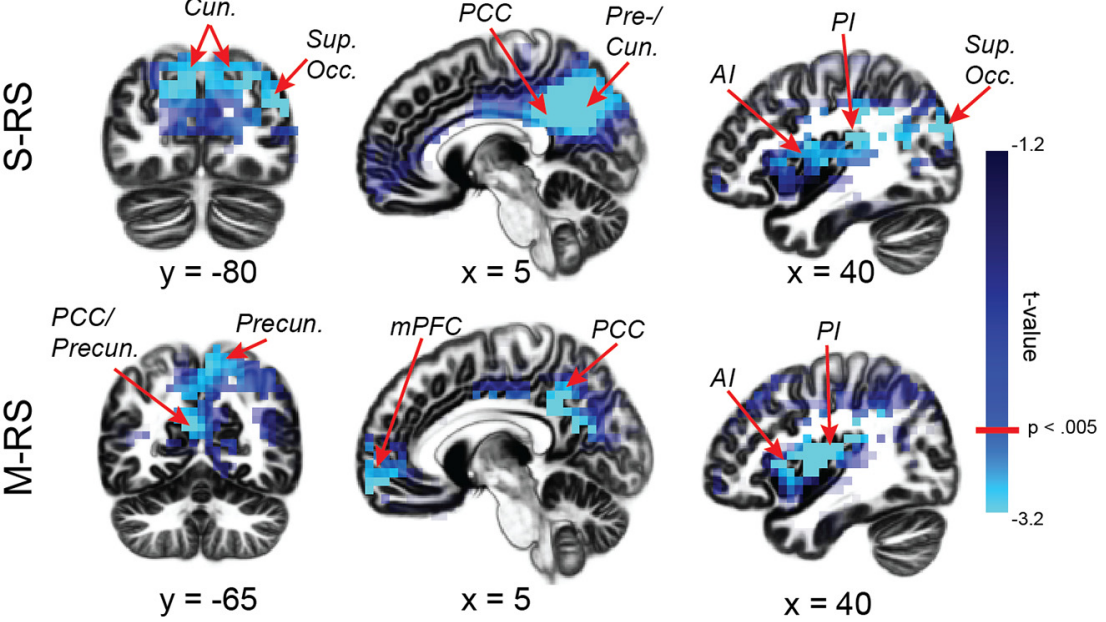

Figure 1. Group differences in $\alpha$ power. A, During the S-RS, the PTSD group demonstrated reduced $\alpha$ power in the VC (the cuneus, precuneus, and superior occipital gyrus), the posterior DMN hub (PCC), and anterior and posterior insula. $\boldsymbol{B}$, During the M-RS, the PTSD group showed reduced $\alpha$ power in the VC (the cuneus and precuneus), both the anterior and posterior DMN hubs (mPFC and $\mathrm{PCC}$ ), and anterior and posterior insula. Cun = cuneus; Precun = precuneus; $\mathrm{Al}=$ anterior insula; $\mathrm{PI}=$ posterior insula; Sup. Occ. = superior occipital gyrus.

$p=0.001$, FDR $p<0.05, d=0.85)$ and $\mathrm{mPFC} \rightarrow \mathrm{PCC}(t=$ $-3.14, p=0.003$, FDR $p<0.05, d=0.76)$, suggesting disrupted DMN connectivity in general by salient visual input (at $\mathrm{M}-\mathrm{RS})$.

Next, simple contrasts of Group for the S-RS revealed reduced $\alpha$ connectivity from the PCC to the VC $(\mathrm{PCC} \rightarrow \mathrm{VC}$ ) in PTSD (vs controls; $t=-2.26, p=0.027$, $d=0.55$; Fig. $2 A$ ), albeit failing FDR correction. No other effects emerged during this state ( $p s>0.34$ ). For the $\mathrm{M}$ RS, the PTSD group (vs controls) demonstrated reduced connectivity within the DMN, including PCC $\rightarrow \mathrm{mPFC}(t=$ $-2.82, p=0.008$, FDR $p<0.05, d=0.69$; Fig. $2 B)$ and, at a trend level, $\mathrm{mPFC} \rightarrow \mathrm{PCC}(t=-1.91, p=0.064, d=0.47)$. The PTSD group demonstrated additional deficits in $\alpha$ connectivity from the VC to both DMN ROls at M-RS: $\mathrm{VC} \rightarrow \mathrm{PCC}(t=-3.06, p=0.004$, FDR $p<0.05, d=0.75)$ and VC $\rightarrow \operatorname{mPFC}(t=-2.05, p=0.049, d=0.50$; albeit not FDR corrected). No effect appeared in the opposite $(\mathrm{DMN} \rightarrow \mathrm{VC})$ direction (ps $>0.12)$. We further explored whole-brain connectivity with the PCC seed during the MRS between the PTSD and control groups (Fig. 3). At the familywise threshold, correcting for the 84 Brodmann areas examined (FWE $p=0.0006$ ), only one additional area emerged with attenuated connectivity from the PCC to the right associative auditory cortex (superior temporal gyrus, Brodmann's area 22; $t=-3.93, p=0.0003$ ) in the
PTSD group at MRS. This effect thus aligned with the VCPCC dysconnectivity in PTSD above.

Double contrasts of State (M-RS minus S-RS) and Group (PTSD vs control) revealed different effects of state (i.e., visual stimulation) on connectivity between the groups. Greater connectivity reduction from S-RS to M-RS appeared in the PTSD (vs control) group in $\mathrm{VC} \rightarrow \mathrm{PCC}(t=-2.22, p=0.032, d=0.54)$ and $\mathrm{VC} \rightarrow \mathrm{mPFC}$ $(t=-2.18, p=0.036, d=0.53)$. Specifically, from S-RS to M-RS, significant connectivity reduction emerged in $\mathrm{VC} \rightarrow \mathrm{PCC}(t=-2.84, p=0.011$, FDR $p<0.05, d=0.69)$ and $\mathrm{VC} \rightarrow \mathrm{mPFC}(t=-3.04, p=0.007$, FDR $p<0.05$, $d=0.74)$ in the PTSD group, but none in the control group (ps $>0.628$ ). These group effects are also summarized in Table 2.

Finally, individual group contrasts were performed to substantiate the PTSD versus controls difference. Again, these specific contrasts revealed consistent results to those from the main analyses (Table 3). A notable difference was that, owing to the reduced group size, the $\mathrm{PCC} \rightarrow \mathrm{VC}$ deficit in PTSD at S-RS survived one-tailed tests only (PTSD vs HC: $t=-1.72, p=0.046$ one-tailed, $d=0.52 ;$ vs GAD: $t=-1.83, p=0.037$ one-tailed, $d=0.53$ ), as did the VC $\rightarrow$ PCC deficit in the double contrast (PTSD vs HC, $t=-1.87, p=0.034$ one-tailed, $d=0.57$; PTSD vs GAD, $t=-1.87, p=0.035$ one-tailed,

Table 2: Summary of group effects

\begin{tabular}{llll}
\hline Effects (PTSD $<$ control) & State & Visual cortex & DMN \\
\hline$\alpha$ Power & S-RS & Cun., Precun., Sup. OcC. & PCC \\
& $M-R S$ & Precun. & PCC, mPFC \\
& $M-R S-S-R S$ & $n . s$. & n. $\rightarrow$ VC \\
$\alpha$ GC & $S-R S$ & $n . s$. & PCC $\rightarrow$ mPFC \\
& $M-R S$ & VC $\rightarrow$ PCC, VC $\rightarrow m P F C$ & $n . s$.
\end{tabular}

Cun = cuneus; Precun = precuneus; Sup. Occ. = superior occipital gyrus; VC = visual cortex. Italicized ones were significant $(p<0.05)$ before multiple comparison correction; all other effects survived correction. 
Table 3: Summary of individual group contrasts

\begin{tabular}{|c|c|c|c|c|}
\hline & Contrast & S-RS & M-RS & M-RS - S-RS \\
\hline \multirow[t]{2}{*}{$\alpha$ Power } & $\begin{array}{c}\text { PTSD vs } \\
\text { HC }\end{array}$ & $\begin{array}{l}\text { PCC/Precun. }(20,-60,35) \\
\quad(t=-3.00 ; k=71) \\
\text { Cun./SOC }(45,-80,25)(t=-3.42 ; \\
\quad k=71) \\
\text { R. Insula }(35,-5,15)(t=-3.18 ; \\
\quad k=10)\end{array}$ & $\begin{array}{l}\text { PCC/Precun. }(5,-55,50)(t=-2.41 ; k=121) \\
\text { mPFC }(10,65,0)(t=-2.87 ; k=10) \\
\text { R. Insula }(45,-5,15)(t=-2.98 ; k=10)\end{array}$ & n.s. \\
\hline & $\begin{array}{c}\text { PTSD vs } \\
\text { GAD }\end{array}$ & $\begin{array}{l}\text { PCC/Precun. }(15,-50,40) \\
\quad(t=-3.31 ; k=112) \\
\text { Cun. }(5,-65,15)(t=-2.88 \\
\quad k=112) \\
\text { L. Insula }(-30,25,0)(t=-4.29 ; \\
\quad k=30)\end{array}$ & $\begin{array}{l}\text { PCC/Precun. }(15,-45,40)(t=-3.31 ; k=102) \\
\text { mPFC }(-15,65,-15)(t=-3.31 ; k=95) \\
\text { R./L. Insula }(60,-15,30 /-30,25,5) \\
\quad(t=-3.96 /-3.58 ; k=64 / 65)\end{array}$ & n.s. \\
\hline$\alpha$ Connectivity & $\begin{array}{l}\text { PTSD vs } \\
\text { HC } \\
\text { PTSD vs } \\
\text { GAD }\end{array}$ & $\begin{array}{l}\mathrm{PCC} \rightarrow \mathrm{VC}^{*}(t=-1.72, p=0.046) \\
\mathrm{PCC} \rightarrow \mathrm{VC}^{*}(t=-1.83, p=0.037)\end{array}$ & $\begin{array}{l}\mathrm{PCC} \rightarrow \mathrm{mPFC}(t=-2.68, p=0.010) \\
\mathrm{VC} \rightarrow \mathrm{PCC}(t=-2.36, p=0.023) \\
\mathrm{PCC} \rightarrow \mathrm{mPFC}(t=-2.68, p=0.010) \\
\mathrm{VC} \rightarrow \mathrm{PCC}(t=-3.30, p=0.002) \\
\mathrm{mPFC} \rightarrow \mathrm{PCC}(t=-3.25, p=0.003)\end{array}$ & $\begin{array}{l}\mathrm{VC} \rightarrow \mathrm{PCC}^{*}(t=-1.87, \\
\quad p=0.034) \\
\mathrm{VC} \rightarrow \mathrm{PCC}^{*}(t=-1.87, \\
p=0.035) \\
\mathrm{VC} \rightarrow \mathrm{mPFC}(t=-2.29, \\
p=0.027)\end{array}$ \\
\hline
\end{tabular}

All effects survived FDR $p<0.05$.

* one-tailed. Peak MNI coordinates $(x, y, z)$ are provided, along with cluster sizes $(\mathrm{k}) . \mathrm{SOC}=$ superior occipital gyrus; $\mathrm{R} / \mathrm{L}=$ right/left.

$d=0.55)$. However, given the strong a priori hypothesis of deficient $\alpha$ connectivity in PTSD, these tests could provide support to the hypothesis. The double contrast on $\mathrm{VC} \rightarrow \mathrm{mPFC} \alpha$ connectivity showed a significant deficit in PTSD in comparison to GAD ( $t=-2.29, p=0.027$, $d=0.67$ ) but only a marginal deficit in comparison to $\mathrm{HC}$ ( $t=-1.50, p=0.071$ one-tailed, $d=0.46$ ).
These group-specific contrasts also revealed additional results: at M-RS, the GAD (vs PTSD or HC) group demonstrated increased $\alpha$ connectivity from $\mathrm{mPFC} \rightarrow \mathrm{PCC}$ (GAD vs PTSD: $t=3.25, p=0.003, d=0.95$; GAD vs HC: $t=2.65$, $p=0.012, d=0.82$ ), suggesting GAD-specific augmentation in this $\alpha$ connectivity. No other differences emerged between the HC and GAD groups (ps > 0.411).

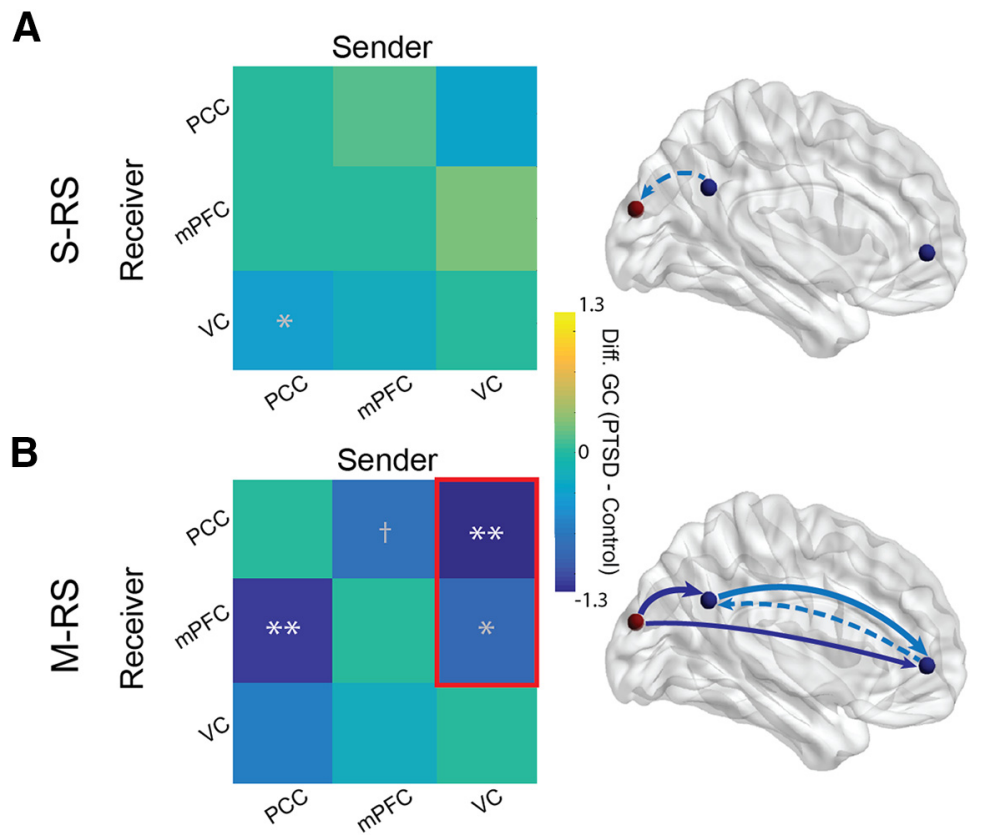

Figure 2. Group differences in $\alpha$ connectivity. Left column, Matrices of group differences PTSD minus controls in directed $\alpha$-frequency connectivity $(\mathrm{GC})$ showed $(\boldsymbol{A})$ reduced $\mathrm{PCC} \rightarrow \mathrm{VC} \alpha$ connectivity (albeit not FDR corrected) during the S-RS; and (B) reduced $\mathrm{PCC} \rightarrow \mathrm{mPFC} \alpha$ connectivity during the M-RS and, as enclosed in a red box, more reduction from the S-RS to the M-RS in $\mathrm{VC} \rightarrow \mathrm{PCC}$ and $\mathrm{VC} \rightarrow \mathrm{mPFC} \alpha$ connectivity. Right column, Schematic presentations of group differences in connectivity during the SRS $(\boldsymbol{A})$ and M-RS $(\boldsymbol{B})$, with solid and dotted arrows reflecting connections surviving and not surviving FDR correction, respectively. Arrows in light blue and dark blue reflect significant effects from simple group contrasts and double contrasts of state and group, respectively. Our discussion focused on the effects surviving the multiple comparison correction; $* p<0.05, * * p<0.01, \dagger p<0.1$; white $*=$ FDR corrected; gray $*=$ not FDR corrected. VC = visual cortex. 


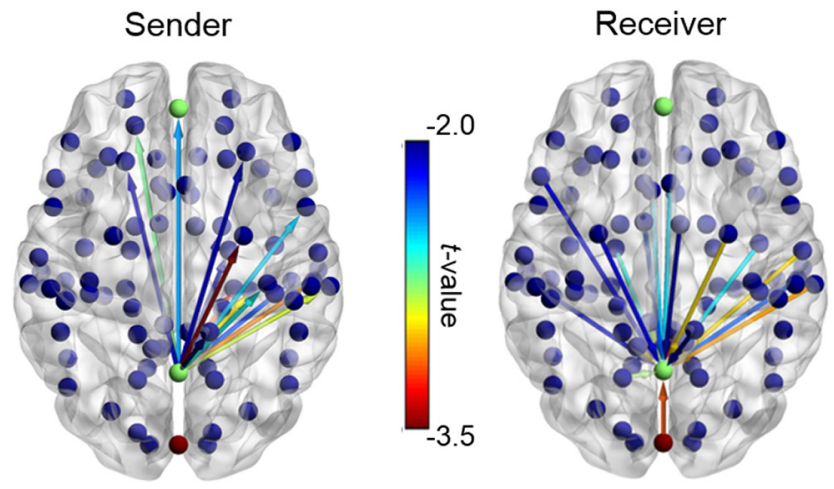

Figure 3. Whole-brain PCC connectivity maps during the MRS. Connectivity for all Brodmann areas (BAs) with the PCC as the sender and receiver $(p<0.05)$. Only the PCC $\rightarrow$ BA 22 connectivity (dark red) survived whole-brain correction (FWE $p=0.0006)$.

\section{Clinical associations}

We then performed correlation analyses, regressing power or connectivity values on symptom severity in hypervigilance. Confirmatory analyses (constrained to the regions identified in the main contrasts) revealed negative associations between hypervigilance and $\alpha$ power in the precuneus/superior parietal lobule at S-RS (39 voxels; peak: $-20,-75,-55 ; r=-0.49, p<0.005$; Fig. $4 A$ ) and the DMN at M-RS: PCC (13 voxels; peak: $5,-45,45 ; r=$ $-0.28, p<0.05)$ and mPFC (50 voxels; peak: $-10,65$, $-15 ; r=-0.30, p<0.05 ;$ Fig. $4 B$ ). Whole-brain regression analysis further revealed negative associations with $\alpha$ power in the ventral VC at S-RS (left inferior temporal gyrus: 13 voxels; peak: $-55,-5,-40 ; r=-0.40$, $p<0.005$ whole-brain corrected; Fig. 4A). There was no significant correlation between hypervigilance and $\alpha$ connectivity ( $p s>0.20$ ).

\section{Discussion}

Source-level analysis of RS $\alpha$ oscillations isolated $\alpha$ (power and connectivity) deficits in the DMN and VC in PTSD, especially during strong visual stimulation. In support of our first hypothesis, $\alpha$ deficits in the DMN, the PTSD group demonstrated reduced $\alpha$ power in the posterior DMN hub (the PCC) at both S-RS and M-RS as well as the anterior hub (the $\mathrm{mPFC}$ ) at M-RS, accompanied by reduced $\mathrm{DMN}$ (PCC $\rightarrow \mathrm{mPFC}) \alpha$-frequency connectivity at M-RS. In support of the second hypothesis, $\alpha$ deficits in the VC, the PTSD group exhibited reduced $\alpha$ power in the VC at both states. Importantly, joining $\alpha$ deficits in the two neural systems, diminished $\alpha$-frequency connectivity from the VC to the DMN was observed in the PTSD group at M-RS. Finally, $\alpha$ power deficits in the DMN and VC directly correlated with symptom severity of hypervigilance. Therefore, linking anomalies in DMN and $\alpha$ activity in PTSD, the current results indicate interrelated $\alpha$ deficits in the DMN and VC, implicating visual-cortex-DMN $\alpha$ dysrhythmia in the neuropathology of PTSD.

Neural oscillations actively participate in mental activities by modulating local neuronal excitability and mediating long-range neural communication (Buzsáki and Draguhn, 2004; Buzsáki et al., 2013). Aberrant restingstate (intrinsic) neural oscillations (e.g., thalamocortical dysrhythmia) in neuropsychiatric disorders has been increasingly recognized (Llinás et al., 1999; Schulman et al., 2011; Vanneste et al., 2018), promoting the transdiagnostic conceptualization of "oscillopathies" for these disorders (Basar, 2013; Buzsáki et al., 2013). Advancements in neural computational algorithms (such as eLORETA) have permitted intracranial source estimation of neural oscillations in hdEEG recordings, providing important insights into oscillatory dysrhythmia in multiple disorders, e.g., schizophrenia (Canuet et al., 2011; Di Lorenzo et al., 2015), depression (Whitton et al., 2018), and PTSD (Imperatori et al., 2014). As validation of our hdEEG source analysis, we confirmed a strong " $\alpha$ blocking" effect of visual stimulation by demonstrating extensive $\alpha$ power reduction in bilateral visual cortices from the S-RS (minimal visual input) to the M-RS (strong visual input). By contrast, no other regions emerged from this contrast, highlighting the sensitivity and specificity of this source analysis of $\alpha$ oscillations.

Our source-level group analysis further identified $\alpha$ deficits within and between the VC and the DMN in PTSD. Concerning the VC, $\alpha$ oscillations are known to mediate visual cortical inhibition, and accordingly, $\alpha$ power correlates inversely with visual cortical activity (Klimesch et al., 2007; Palva and Palva, 2007; Jensen and Mazaheri, 2010; Lange et al., 2013). In the PTSD group, reduced $\alpha$ power in the VC was extensive and enduring across states.
A

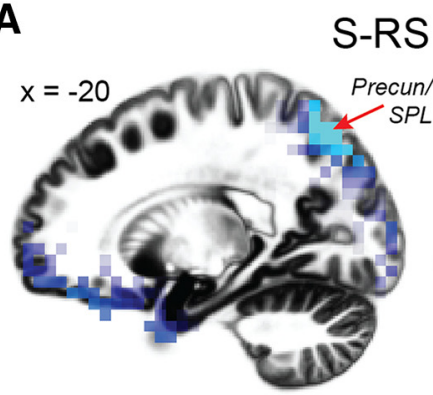

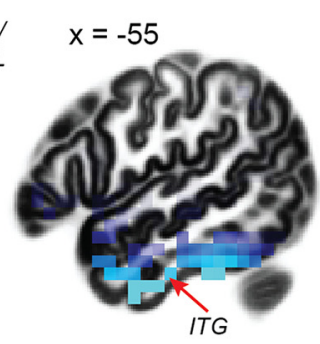

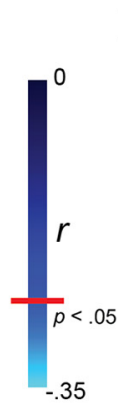

B
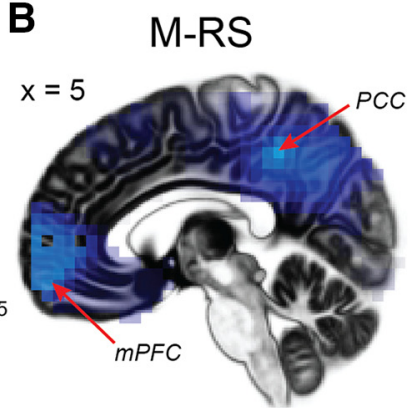

Figure 4. Clinical associations between $\alpha$ power and hypervigilance. Whole-brain correlation maps of $\alpha$ power and hypervigilance indicated negative correlations in both the dorsal (i.e., SPL, precuneus) and ventral (i.e., ITG) visual cortices during the S-RS (A) and both DMN hubs (mPFC and PCC) during the M-RS (B). SPL = superior parietal lobule; ITG = inferior temporal gyrus. 
Without visual stimulation (at S-RS, reflecting intrinsic activity), $\alpha$ deficits spanned a large cluster over the cuneus, precuneus, and superior occipital gyrus, suggesting wide-spread, intrinsic neural disinhibition and hyperactivity across primary and secondary visual cortices in PTSD. This sensory cortical disinhibition aligns with extant electrophysiological evidence of impaired sensory gating and sensory cortical hyperactivity (to simple, neutral stimuli; Morgan and Grillon, 1999; Neylan et al., 1999; Stewart and White, 2008; Javanbakht et al., 2011) and behavioral disturbances in sensory filtering/gating and response in these patients (Stewart and White, 2008; Engel-Yeger et al., 2013). With strong visual input at M-RS, $\alpha$ power reduction was particularly localized to the parietal VC (primarily bilateral precuneus). Given that this region is strongly involved in visual spatial attention and perception (Corbetta et al., 1995; Behrmann et al., 2004; Corbetta and Shulman, 2011) and that the M-RS condition mimics a real-life environment with salient sensory information, this $\alpha$ deficit (reflective of disinhibited visual spatial attention) can underlie hypervigilance in PTSD, expressed as excessive alertness to and scanning of the environment (Conoscenti et al., 2009).

As mentioned above, $\alpha$ oscillations also play a role in long-range neural communication and resting-state $\alpha$ power correlates positively with DMN activity (Mantini et al., 2007; Jann et al., 2009; Sadaghiani et al., 2010; Knyazev et al., 2011; Mo et al., 2013; Samogin et al., 2019). The $\alpha$ deficits in DMN hubs thus dovetails the extant literature citing both deficient $\alpha$ activity (Clancy et al., 2017; Clancy et al., 2020) and DMN hypoactivity in PTSD (Koch et al., 2016; Akiki et al., 2018). Notably, restingstate $\beta$ and $\theta$ oscillations have also been found to be associated with DMN activity (Laufs et al., 2003; Mantini et al., 2007; Scheeringa et al., 2012). However, prior sensorlevel analyses have not revealed PTSD-related anomalies in the $\beta$ or $\theta$ frequencies (ps $>0.16$; Clancy et al., 2017). Future research is warranted to examine the other oscillatory activities in various states or tasks to elucidate their contribution to PTSD pathology.

The fact that DMN $\alpha$ power reduction extended from the PCC only at S-RS to both the PCC and MPFC at MRS accentuates the particular DMN vulnerability in PTSD in a sensory-rich environment. In addition, as $\alpha$ oscillations synchronize activity and facilitate coherence across regions, reduced $\alpha$ power in these key DMN hubs could further suggest compromised communication across the network in PTSD. Indeed, connectivity analyses revealed reduced $\mathrm{PCC} \rightarrow \mathrm{mPFC} \alpha$ connectivity at M-RS in the PTSD group. This hypoconnectivity between the DMN hubs highlights impaired communication within the core architecture of the DMN. Clinically, DMN $\alpha$ deficits in both local power and interhub connectivity, especially acute in a sensory-rich environment, could contribute to difficulty in maintaining "tranquility" or "rest" (Buckner et al., 2008; Abdallah et al., 2017; Akiki et al., 2018) and avoidance of sensory stimulation in patients with PTSD (Stewart and White, 2008; Engel-Yeger et al., 2013).

Our manipulation of visual stimulation between the two states revealed a direct link between $\alpha$ deficits in the VC and the DMN. Consistent with general DMN susceptibility to salient sensory input, we confirmed a general reduction (in the entire sample) in bidirectional $\alpha$ connectivity between the DMN hubs (PCC $\rightarrow \mathrm{mPFC}$ and $\mathrm{mPFC} \rightarrow \mathrm{PC}$ ) as visual input increased from the S-RS to the M-RS. Beyond that, in the PTSD (but not control) group, increased visual stimulation (from S-RS to M-RS) further diminished visual cortical $\alpha$ connectivity to both DMN hubs $(\mathrm{VC} \rightarrow \mathrm{PCC}$ and $\mathrm{VC} \rightarrow \mathrm{mPFC}$ ). As mentioned above, $\alpha$ oscillations in the sensory cortex mediate sensory inhibition, such that this $\mathrm{VC} \rightarrow \mathrm{DMN} \alpha$ projection would serve to protect the DMN by blocking sensory afferents to the network. This notion is supported by prior work demonstrating a modulatory role of $\alpha$ oscillations in the connectivity within the sensory (visual) cortex and between the VC and DMN hubs (Scheeringa et al., 2012). While this protective inhibitory process withstood the increased visual input at M-RS in the control group, it broke down among patients with PTSD, suggesting impaired gating of sensory entry to (i.e., compromised protection of) the DMN. Exploratory correlation analyses further indicated a close correlation between $\mathrm{VC} \rightarrow \mathrm{PCC}$ and $\mathrm{PCC} \rightarrow \mathrm{mPFC} \alpha$ connectivity at M-RS $(r=0.31, p=0.013)$, highlighting a mechanistic link between these two pathways. That is, disinhibited visual cortical propagation to the PCC, in the presence of strong environmental input, could further impair PCCdriven $\alpha$ synchronization with the mPFC, worsening DMN dysfunction in PTSD. Finally, to rule out the alternative explanation that emotional content (beyond visual stimulation) could contribute to the M-RS effects, we compared RS EEG data acquired before and after 5min presentation of negative international affective picture system (IAPS) pictures from an independent sample of healthy individuals $(N=45)$. There was no change in $\alpha \mathrm{GC}(t=0.032, p=0.974)$, suggesting that in the absence of visual stimulation, simple affective effects would not result in $\alpha$ connectivity change. Nonetheless, we cannot fully exclude the combined effects of emotion and visual stimulation. That is, the emotional content of visual stimuli may influence $\alpha$ GC differently among the three groups. Future studies are warranted to isolate the simple effects of visual stimulation by including neutral images alone.

Together, current findings implicate a core visual-cortex-DMN system of $\alpha$ dysrhythmia in PTSD. The critical role of visual cortical $\alpha$ deficits in this pathology lends credence to a sensory hypothesis of PTSD centered on sensory cortical disinhibition (Clancy et al., 2017, 2020; Li, 2019) and bottom-up accounts of PTSD in general (Nicholson et al., 2017; Badura-Brack et al., 2018). Childhood trauma, a common PTSD risk factor, has been associated with various aberrations in the sensory cortex and sensory pathway (Teicher et al., 2016), adding to the support for this sensory hypothesis. Indeed, $36 \%$ of the current PTSD sample reported childhood trauma. That said, the means (and standard deviations) of $\alpha$ power and connectivity were highly comparable between the childhood trauma subgroup and the rest of the PTSD group, implicating this sensory pathology across trauma types. 
This core visual-cortex-DMN system of $\alpha$ dysrhythmia was reinforced by the specificity of $\alpha$ deficit sources. Despite the use of whole-brain analyses, group differences in $\alpha$ power and clinical associations between $\alpha$ power and hypervigilance were localized to the $\mathrm{VC}$ and the DMN only (except for the associative auditory cortex and insula as discussed below). While DMN hypoactivity is known to be associated with PTSD symptom severity (Sripada et al., 2012; Miller et al., 2017a,b; Akiki et al., 2018), the clinical association in the VC highlights an additional pathway linking visual cortical disinhibition to PTSD symptoms. Notably, the associations with hypervigilance implicate not only both the dorsal (i.e., the parietal cortex) and ventral visual cortices (i.e., the inferior temporal gyrus) but also the higher-order regions with strong interactions with limbic and frontal regions. As the ventral VC underpins visual object perception, its involvement here aligns with the fact that hypervigilance in PTSD is associated with hyperactivity and hypersensitivity to (threat and neutral) sensory cues (Ehlers and Clark, 2000; Hayes et al., 2012), in addition to excessive spatial scanning engaging the dorsal VC. In light of the "sentinel hypothesis" implicating the DMN in sustained monitoring of the environment (i.e., sensory vigilance; Buckner et al., 2008; Andrews-Hanna, 2012), we surmise this visual-cortex-DMN $\alpha$ dysrhythmia in PTSD may fuse the impairment in "sentinel" function (of the DMN) and in sensory cortical inhibition (via $\alpha$ oscillations), resulting in a pathologic state hypervigilance.

While the insula was not an a priori ROI in the current study, expansive $\alpha$ deficits spanning the posterior and anterior insula emerged in PTSD following whole-brain correction. The insula is a highly heterogeneous structure, with the posterior portion receiving strong sensory afferents and the anterior portion densely connected with limbic and prefrontal regions as a key node of the salience network/SN (Craig, 2002; Critchley, 2004; Seeley et al., 2007; Menon and Uddin, 2010). Abnormal insular and SN activity has been repeatedly observed in patients with PTSD (Lanius et al., 2015; Koch et al., 2016), and $\alpha$ suppression via neurofeedback can increase SN activity (Ros et al., 2013). Our data provide preliminary evidence of insular $\alpha$ dysrhythmia in PTSD, potentially underlying some of the insular anomalies. Interestingly, our exploratory whole-brain connectivity analyses further isolated an additional region, the right superior temporal gyrus, showing attenuated connectivity with the DMN (i.e., PCC) in PTSD. This area represents the associative auditory cortex and a primary source of auditory $\alpha$ ("tau") oscillations (Lehtelä et al., 1997; Billig et al., 2019). This finding thus further bolsters the pathology of sensory-cortex-DMN $\alpha$ dysconnectivity in PTSD, extending beyond our a priori focus on the VC to other sensory modalities.

We included patients with GAD as an additional control condition to rule out general effects of anxiety and arousal on $\alpha$ activity. Previous sensor-level analysis of $\alpha$ oscillations revealed no $\alpha$ deficits in GAD (Clancy et al., 2017), which directed us to combine patients with GAD and HCs into one control group in the source-level analysis. Nonetheless, we systematically explored group-specific effects. While the results essentially echoed the main results, we noticed interesting findings of enhanced DMN $(\mathrm{mPFC} \rightarrow \mathrm{PCC}) \alpha$ connectivity in GAD at M-RS, in comparison to the PTSD and HC groups. We surmise that this $\mathrm{mPFC} \rightarrow \mathrm{PCC}$ hyper-connectivity could heighten DMN functioning in GAD, supporting the hallmark symptoms of self-referential rumination and worry in these patients.

Integrative neuroimaging and electrophysiological research have promoted the idea that $\alpha$ oscillations sustain and facilitate DMN functioning by synchronizing spontaneous activity across the network and suppressing sensory cortical propagation. Translating these mechanisms to the neuropathology of PTSD, we confirmed interconnected $\alpha$ deficits in the DMN and VC in patients with PTSD, which are further associated with symptoms of hypervigilance. Therefore, the current findings provide the first evidence of visual-cortex-DMN $\alpha$ dysrhythmia in PTSD, presenting a unifying neural underpinning of sensory disinhibition, DMN dysfunction, and hypervigilance in this disorder. The specification of this $\alpha$ dysrhythmia further isolates a novel therapeutic target, promoting network-based interventions (Lanius et al., 2015) using brain stimulation of $\alpha$ oscillations (Clancy et al., 2018) in the visual-cortex-DMN system as a new line of treatment for PTSD.

\section{References}

Abdallah CG, Southwick SM, Krystal JH (2017) Neurobiology of posttraumatic stress disorder (PTSD): a path from novel pathophysiology to innovative therapeutics. Neurosci Lett 649:130-132.

Akiki TJ, Averill CL, Abdallah CG (2017) A network-based neurobiological model of PTSD: evidence from structural and functional neuroimaging studies. Curr Psychiatry Rep 19:81.

Akiki TJ, Averill CL, Wrocklage KM, Scott JC, Averill LA, Schweinsburg B, Alexander-Bloch A, Martini B, Southwick SM, Krystal JH, Abdallah CG (2018) Default mode network abnormalities in posttraumatic stress disorder: a novel network-restricted topology approach. Neuroimage 176:489-498.

American Psychiatric Association (2013) Diagnostic and statistical manual of mental disorders, Ed 5. Washington, DC: American Psychiatric Association.

Andrews-Hanna JR (2012) The brain's default network and its adaptive role in internal mentation. Neuroscientist 18:251-270.

Badura-Brack A, McDermott TJ, Heinrichs-Graham E, Ryan TJ, Khanna MM, Pine DS, Bar-Haim Y, Wilson TW (2018) Veterans with PTSD demonstrate amygdala hyperactivity while viewing threatening faces: a MEG study. Biol Psychol 132:228-232.

Basar E (2013) Brain oscillations in neuropsychiatric disease. Dialogues Clin Neurosci 15:291-300.

Behrmann M, Geng JJ, Shomstein S (2004) Parietal cortex and attention. Curr Opin Neurobiol 14:212-217.

Billig AJ, Herrmann B, Rhone AE, Gander PE, Nourski KV, Snoad BF, Kovach CK, Kawasaki H, Howard MA 3rd, Johnsrude IS (2019) A sound-sensitive source of alpha oscillations in human non-primary auditory cortex. J Neurosci 39:8679-8689.

Blanchard EB, Jones-Alexander J, Buckley TC, Forneris CA (1996) Psychometric properties of the PTSD checklist (PCL). Behav Res Ther 34:669-673.

Bluhm RL, Williamson PC, Osuch EA, Frewen PA, Stevens TK, Boksman K, Neufeld RW, Théberge J, Lanius RA (2009) Alterations in default network connectivity in posttraumatic stress disorder related to early-life trauma. J Psychiatry Neurosci 34:187-194. 
Buckner RL, Andrews-Hanna JR, Schacter DL (2008) The brain's default network: anatomy, function, and relevance to disease. Ann NY Acad Sci 1124:1-38.

Buzsáki G, Draguhn A (2004) Neuronal oscillations in cortical networks. Science 304:1926-1929.

Buzsáki G, Logothetis N, Singer W (2013) Scaling brain size, keeping timing: evolutionary preservation of brain rhythms. Neuron 80:751-764

Canuet L, Ishii R, Pascual-Marqui RD, Iwase M, Kurimoto R, Aoki Y, Ikeda S, Takahashi H, Nakahachi T, Takeda M (2011) Restingstate EEG source localization and functional connectivity in schizophrenia-like psychosis of epilepsy. PLoS One 6:e27863.

Clancy K, Ding M, Bernat E, Schmidt NB, Li W (2017) Restless 'rest': intrinsic sensory hyperactivity and disinhibition in post-traumatic stress disorder. Brain 140:2041-2050.

Clancy KJ, Baisley SK, Albizu A, Kartvelishvili N, Ding M, Li W (2018) Lasting connectivity increase and anxiety reduction via transcranial alternating current stimulation. Soc Cogn Affect Neurosci 13:1305-1316.

Clancy KJ, Albizu A, Schmidt NB, Li W (2020) Intrinsic sensory disinhibition contributes to intrusive re-experiencing in combat veterans. Sci Rep 10:936.

Conoscenti L, Vine V, Papa A, Litz B (2009) Scanning for danger: readjustment to the noncombat environment. In: Living and surviving in harm's way: a psychological treatment handbook for pre-and post-deployment of military personnel, pp 123-145. New York: Routledge.

Corbetta M, Shulman GL (2011) Spatial neglect and attention networks. Annu Rev Neurosci 34:569-599.

Corbetta M, Shulman GL, Miezin FM, Petersen SE (1995) Superior parietal cortex activation during spatial attention shifts and visual feature conjunction. Science 270:802-805.

Craig AD (2002) How do you feel? Interoception: the sense of the physiological condition of the body. Nat Rev Neurosci 3:655-666.

Critchley HD (2004) The human cortex responds to an interoceptive challenge. Proc Natl Acad Sci USA 101:6333-6334.

Dierks T, Jelic V, Pascual-Marqui RD, Wahlund L, Julin P, Linden DE, Maurer K, Winblad B, Nordberg A (2000) Spatial pattern of cerebral glucose metabolism (PET) correlates with localization of intracerebral EEG-generators in Alzheimer's disease. Clin Neurophysiol 111:1817-1824.

Di Lorenzo G, Daverio A, Ferrentino F, Santarnecchi E, Ciabattini F, Monaco L, Lisi G, Barone Y, Di Lorenzo C, Niolu C, Seri S, Siracusano A (2015) Altered resting-state EEG source functional connectivity in schizophrenia: the effect of illness duration. Front Hum Neurosci 9:234.

Ding M, Chen Y, Bressler SL (2006) Granger causality: basic theory and application to neuroscience. In: Handbook of time series analysis, pp 437-460. Weinheim: Wiley-VCH Verlag GmbH \& Co. KGaA.

Ehlers A, Clark DM (2000) A cognitive model of posttraumatic stress disorder. Behav Res Ther 38:319-345.

Engel AK, Singer W (2001) Temporal binding and the neural correlates of sensory awareness. Trends Cogn Sci 5:16-25.

Engel-Yeger B, Palgy-Levin D, Lev-Wiesel R (2013) The sensory profile of people with post-traumatic stress symptoms. Occup Ther Mental Health 29:266-278.

Foxe JJ, Snyder AC (2011) The role of alpha-band brain oscillations as a sensory suppression mechanism during selective attention. Front Psychol 2:154.

Greicius MD, Menon V (2004) Default-mode activity during a passive sensory task: uncoupled from deactivation but impacting activation. J Cogn Neurosci 16:1484-1492.

Greicius MD, Krasnow B, Reiss AL, Menon V (2003) Functional connectivity in the resting brain: a network analysis of the default mode hypothesis. Proc Natl Acad Sci USA 100:253-258.

Gusnard DA, Akbudak E, Shulman GL, Raichle ME (2001) Medial prefrontal cortex and self-referential mental activity: relation to a default mode of brain function. Proc Natl Acad Sci USA 98:42594264.
Hayes JP, Vanelzakker MB, Shin LM (2012) Emotion and cognition interactions in PTSD: a review of neurocognitive and neuroimaging studies. Front Integr Neurosci 6:89.

Hermans EJ, Henckens MJ, Joëls M, Fernández G (2014) Dynamic adaptation of large-scale brain networks in response to acute stressors. Trends Neurosci 37:304-314.

Hillebrand A, Tewarie P, van Dellen E, Yu M, Carbo EW, Douw L, Gouw AA, van Straaten EC, Stam CJ (2016) Direction of information flow in large-scale resting-state networks is frequency-dependent. Proc Natl Acad Sci USA 113:3867-3872.

Imperatori C, Farina B, Quintiliani MI, Onofri A, Castelli Gattinara P, Lepore M, Gnoni V, Mazzucchi E, Contardi A, Della Marca G (2014) Aberrant EEG functional connectivity and EEG power spectra in resting state post-traumatic stress disorder: a sLORETA study. Biol Psychol 102:10-17.

Imperatori C, Farina B, Adenzato M, Valenti EM, Murgia C, Marca GD, Brunetti R, Fontana E, Ardito RB (2019) Default mode network alterations in individuals with high-trait-anxiety: an EEG functional connectivity study. J Affect Disord 246:611-618.

Jann K, Dierks T, Boesch C, Kottlow M, Strik W, Koenig T (2009) BOLD correlates of EEG alpha phase-locking and the fMRI default mode network. Neuroimage 45:903-916.

Javanbakht A, Liberzon I, Amirsadri A, Gjini K, Boutros NN (2011) Event-related potential studies of post-traumatic stress disorder: a critical review and synthesis. Biol Mood Anxiety Disord 1:5.

Jensen O, Mazaheri A (2010) Shaping functional architecture by oscillatory alpha activity: gating by inhibition. Front Hum Neurosci 4:186.

Johnson EL, Dewar CD, Solbakk AK, Endestad T, Meling TR, Knight RT (2017) Bidirectional Frontoparietal Oscillatory Systems Support Working Memory. Curr Biol 27:1829-1835.e4.

Klimesch W (2012) alpha-band oscillations, attention, and controlled access to stored information. Trends Cogn Sci 16:606-617.

Klimesch W, Sauseng P, Hanslmayr S (2007) EEG alpha oscillations: the inhibition-timing hypothesis. Brain Res Rev 53:63-88.

Knyazev GG, Slobodskoj-Plusnin JY, Bocharov AV, Pylkova LV (2011) The default mode network and EEG $\alpha$ oscillations: an independent component analysis. Brain Res 1402:67-79.

Koch SB, van Zuiden M, Nawijn L, Frijling JL, Veltman DJ, Olff M (2016) Aberrant resting-state brain activity in posttraumatic stress disorder: a meta-analysis and systematic review. Depress Anxiety 33:592-605.

Lang P, Bradley M, Cuthbert BN (2008) International affective picture system (IAPS): Affective ratings of pictures and instruction manual. Technical Report A-8. University of Florida, Gainesville, FL.

Lange J, Oostenveld R, Fries P (2013) Reduced occipital alpha power indexes enhanced excitability rather than improved visual perception. J Neurosci 33:3212-3220.

Lanius RA, Bluhm RL, Coupland NJ, Hegadoren KM, Rowe B, Théberge J, Neufeld RW, Williamson PC, Brimson M (2010) Default mode network connectivity as a predictor of post-traumatic stress disorder symptom severity in acutely traumatized subjects. Acta Psychiatr Scand 121:33-40.

Lanius RA, Frewen PA, Tursich M, Jetly R, McKinnon MC (2015) Restoring large-scale brain networks in PTSD and related disorders: a proposal for neuroscientifically-informed treatment interventions. Eur J Psychotraumatol 6:27313.

Laufs H, Krakow K, Sterzer P, Eger E, Beyerle A, Salek-Haddadi A, Kleinschmidt A (2003) Electroencephalographic signatures of attentional and cognitive default modes in spontaneous brain activity fluctuations at rest. Proc Natl Acad Sci USA 100:11053-11058.

Lehtelä L, Salmelin R, Hari R (1997) Evidence for reactive magnetic $10-\mathrm{Hz}$ rhythm in the human auditory cortex. Neurosci Lett 222:111-114.

Li W (2019) Perceptual mechanisms of anxiety and its disorders. In: Cambridge handbook of anxiety and related disorders (Olatunji B, ed). Cambridge: Cambridge University Press.

Liberzon I, Abelson JL (2016) Context processing and the neurobiology of post-traumatic stress disorder. Neuron 92:14-30. 
Liu Q, Farahibozorg S, Porcaro C, Wenderoth N, Mantini D (2017) Detecting large-scale networks in the human brain using high-density electroencephalography. Hum Brain Mapp 38:4631-4643.

Llinás RR, Ribary U, Jeanmonod D, Kronberg E, Mitra PP (1999) Thalamocortical dysrhythmia: a neurological and neuropsychiatric syndrome characterized by magnetoencephalography. Proc Natl Acad Sci USA 96:15222-15227.

Mantini D, Perrucci MG, Del Gratta C, Romani GL, Corbetta M (2007) Electrophysiological signatures of resting state networks in the human brain. Proc Natl Acad Sci USA 104:13170-13175.

Menon V (2011) Large-scale brain networks and psychopathology: a unifying triple network model. Trends Cogn Sci 15:483-506.

Menon V, Uddin LQ (2010) Saliency, switching, attention and control: a network model of insula function. Brain Struct Funct 214:655-667.

Miller DR, Hayes SM, Hayes JP, Spielberg JM, Lafleche G, Verfaellie M (2017a) Default mode network subsystems are differentially disrupted in posttraumatic stress disorder. Biol Psychiatry Cogn Neurosci Neuroimaging 2:363-371.

Miller DR, Logue MW, Wolf EJ, Maniates H, Robinson ME, Hayes JP, Stone A, Schichman S, McGlinchey RE, Milberg WP, Miller MW (2017b) Posttraumatic stress disorder symptom severity is associated with reduced default mode network connectivity in individuals with elevated genetic risk for psychopathology. Depress Anxiety 34:632-640.

Mo J, Liu Y, Huang H, Ding M (2013) Coupling between visual alpha oscillations and default mode activity. Neuroimage 68:112-118.

Mobascher A, Brinkmeyer J, Warbrick T, Musso F, Wittsack HJ, Stoermer R, Saleh A, Schnitzler A, Winterer G (2009) Fluctuations in electrodermal activity reveal variations in single trial brain responses to painful laser stimuli-a fMRI/EEG study. Neuroimage 44:1081-1092.

Morgan CA 3rd, Grillon C (1999) Abnormal mismatch negativity in women with sexual assault-related posttraumatic stress disorder. Biol Psychiatry 45:827-832.

Mueller-Pfeiffer C, Schick M, Schulte-Vels T, O'Gorman R, Michels L, Martin-Soelch C, Blair JR, Rufer M, Schnyder U, Zeffiro T, Hasler G (2013) Atypical visual processing in posttraumatic stress disorder. Neuroimage Clin 3:531-538.

Mulert C, Jäger L, Schmitt R, Bussfeld P, Pogarell O, Moller HJ, Juckel G, Hegerl U (2004) Integration of fMRI and simultaneous EEG: towards a comprehensive understanding of localization and timecourse of brain activity in target detection. Neuroimage 22:83-94.

Neuner I, Arrubla J, Werner CJ, Hitz K, Boers F, Kawohl W, Shah NJ (2014) The default mode network and EEG regional spectral power: a simultaneous fMRI-EEG study. PLoS One 9:e88214.

Neylan TC, Fletcher DJ, Lenoci M, McCallin K, Weiss DS, Schoenfeld FB, Marmar CR, Fein G (1999) Sensory gating in chronic posttraumatic stress disorder: reduced auditory P50 suppression in combat veterans. Biol Psychiatry 46:1656-1664.

Nicholson AA, Friston KJ, Zeidman P, Harricharan S, McKinnon MC, Densmore M, Neufeld RWJ, Théberge J, Corrigan F, Jetly R, Spiegel D, Lanius RA (2017) Dynamic causal modeling in PTSD and its dissociative subtype: bottom-up versus top-down processing within fear and emotion regulation circuitry. Hum Brain Mapp 38:5551-5561.

Nolan H, Whelan R, Reilly RB (2010) FASTER: fully automated statistical thresholding for EEG artifact rejection. J Neurosci Methods 192:152-162.

Olbrich S, Mulert C, Karch S, Trenner M, Leicht G, Pogarell O, Hegerl $U$ (2009) EEG-vigilance and BOLD effect during simultaneous EEG/fMRI measurement. Neuroimage 45:319-332.

Palva S, Palva JM (2007) New vistas for alpha-frequency band oscillations. Trends Neurosci 30:150-158.

Pascual-Marqui RD, Lehmann D, Koukkou M, Kochi K, Anderer P, Saletu B, Tanaka H, Hirata K, John ER, Prichep L, Biscay-Lirio R, Kinoshita T (2011) Assessing interactions in the brain with exact low-resolution electromagnetic tomography. Philos Trans A Math Phys Eng Sci 369:3768-3784.

Pizzagalli DA, Oakes TR, Fox AS, Chung MK, Larson CL, Abercrombie HC, Schaefer SM, Benca RM, Davidson RJ (2004)
Functional but not structural subgenual prefrontal cortex abnormalities in melancholia. Mol Psychiatry 9:325, 393-405.

Raichle ME, MacLeod AM, Snyder AZ, Powers WJ, Gusnard DA, Shulman GL (2001) A default mode of brain function. Proc Natl Acad Sci USA 98:676-682.

Rauch SL, Shin LM, Phelps EA (2006) Neurocircuitry models of posttraumatic stress disorder and extinction: human neuroimaging research-past, present, and future. Biol Psychiatry 60:376-382.

Ros T, Théberge J, Frewen PA, Kluetsch R, Densmore M, Calhoun VD, Lanius RA (2013) Mind over chatter: plastic up-regulation of the fMRI salience network directly after EEG neurofeedback. Neuroimage 65:324-335.

Sadaghiani S, Scheeringa R, Lehongre K, Morillon B, Giraud AL, Kleinschmidt A (2010) Intrinsic connectivity networks, alpha oscillations, and tonic alertness: a simultaneous electroencephalography/functional magnetic resonance imaging study. J Neurosci 30:10243-10250.

Samogin J, Liu Q, Marino M, Wenderoth N, Mantini D (2019) Shared and connection-specific intrinsic interactions in the default mode network. Neuroimage 200:474-481.

Scheeringa $R$, Petersson KM, Kleinschmidt A, Jensen $O$, Bastiaansen MCM (2012) EEG $\alpha$ power modulation of fMRI resting-state connectivity. Brain Connect 2:254-264.

Schulman JJ, Cancro R, Lowe S, Lu F, Walton KD, Llinás RR (2011) Imaging of thalamocortical dysrhythmia in neuropsychiatry. Front Hum Neurosci 5:69.

Seeley WW, Menon V, Schatzberg AF, Keller J, Glover GH, Kenna H, Reiss AL, Greicius MD (2007) Dissociable intrinsic connectivity networks for salience processing and executive control. J Neurosci 27:2349-2356.

Spielberg JM, McGlinchey RE, Milberg WP, Salat DH (2015) Brain network disturbance related to posttraumatic stress and traumatic brain injury in veterans. Biol Psychiatry 78:210-216.

Sripada RK, King AP, Welsh RC, Garfinkel SN, Wang X, Sripada CS, Liberzon I (2012) Neural dysregulation in posttraumatic stress disorder: evidence for disrupted equilibrium between salience and default mode brain networks. Psychosom Med 74:904-911.

Stewart LP, White PM (2008) Sensory filtering phenomenology in PTSD. Depress Anxiety 25:38-45.

Tamber-Rosenau BJ, Dux PE, Tombu MN, Asplund CL, Marois R (2013) Amodal processing in human prefrontal cortex. J Neurosci 33:11573-11587.

Tang AC, Sutherland MT, Sun P, Zhang Y, Nakazawa M, Korzekwa A, Yang Z, Ding M (2007) Top-down versus bottom-up processing in the human brain: distinct directional influences revealed by integrating SOBI and Granger causality. In: Independent component analysis and signal separation: 7th international conference, ICA 2007, London, UK, September 9-12, 2007. Proceedings (Davies ME, James CJ, Abdallah SA, Plumbley MD, eds), pp 802-809. Berlin; Heidelberg: Springer Berlin Heidelberg.

Teicher MH, Samson JA, Anderson CM, Ohashi K (2016) The effects of childhood maltreatment on brain structure, function and connectivity. Nat Rev Neurosci 17:652-666.

Thatcher RW, North DM, Biver CJ (2014) LORETA EEG phase reset of the default mode network. Front Hum Neurosci 8:529.

Uhlhaas PJ, Haenschel C, Nikolić D, Singer W (2008) The role of oscillations and synchrony in cortical networks and their putative relevance for the pathophysiology of schizophrenia. Schizophr Bull 34:927-943.

Vanneste S, Song JJ, De Ridder D (2018) Thalamocortical dysrhythmia detected by machine learning. Nat Commun 9:1103.

Vitacco D, Brandeis D, Pascual-Marqui R, Martin E (2002) Correspondence of event-related potential tomography and functional magnetic resonance imaging during language processing. Hum Brain Mapp 17:4-12.

Wang C, Rajagovindan R, Han SM, Ding M (2016) Top-down control of visual alpha oscillations: sources of control signals and their mechanisms of action. Front Hum Neurosci 10:15.

Whitton AE, Deccy S, Ironside ML, Kumar P, Beltzer M, Pizzagalli DA (2018) Electroencephalography source functional connectivity 
reveals abnormal high-frequency communication among largescale functional networks in depression. Biol Psychiatry Cogn Neurosci Neuroimaging 3:50-58.

Worrell GA, Lagerlund TD, Sharbrough FW, Brinkmann BH, Busacker NE, Cicora KM, O'Brien TJ (2000) Localization of the epileptic focus by low-resolution electromagnetic tomography in patients with a lesion demonstrated by MRI. Brain Topogr 12:273282.

Yeo BT, Krienen FM, Sepulcre J, Sabuncu MR, Lashkari D, Hollinshead M, Roffman JL, Smoller JW, Zöllei L, Polimeni JR,
Fischl B, Liu H, Buckner RL (2011) The organization of the human cerebral cortex estimated by intrinsic functional connectivity. J Neurophysiol 106:1125-1165.

Zhang W, Hashemi MM, Kaldewaij R, Koch SBJ, Beckmann C, Klumpers F, Roelofs K (2019) Acute stress alters the 'default' brain processing. Neuroimage 189:870-877.

Zumsteg D, Friedman A, Wennberg RA, Wieser HG (2005) Source localization of mesial temporal interictal epileptiform discharges: correlation with intracranial foramen ovale electrode recordings. Clin Neurophysiol 116:2810-2818. 